$35 \mathrm{pts}$ ); MTX plus prednisolone (P) $10 \mathrm{mg}$ daily (MTX-P, $34 \mathrm{pts}$ ); MTX-P plus methylprednisolone (MP) $1000 \mathrm{mg}$ intravenously on the first day of treatment (MTX-P-MP, $35 \mathrm{pts}$ ); leflunomide $20 \mathrm{mg}$ daily (LEF, $37 \mathrm{pts}$ ). Duration of treatment was one year. Control points were 3,6 and 12 months from the initiation of therapy. Safety data was assessed at the main control points.

Results: One hundred twenty-seven pts completed the study. Side effects were registered in the same number of patients in each group ( 9 patients; $24,3 \%>26 \%$ ). Therapy had to be stopped in six patients due to side effects: MTX - 1 (depigmentation of the skin), MTX-P - none, MTX-P-MP - 1 (stomatitis) and LEF - 4 (dermatitis-2; pancytopenia with platelet count $43 \times 10^{9} / \mathrm{L}$, erythrocyte $2,9 \times 10^{12} / \mathrm{L}, \mathrm{WBC} 2 \times 10^{9} / \mathrm{L}-1$; angioedema, periorbital edema and dermatitis with itching-1). Other side effects were mild: MTX - 8 pts (dyspepsia-1, elevation of transaminases-6, hair loss-1), MTX-P - 9 pts (Cushing's syndrome - 1, hair loss - 1 , anemia - 1 , elevation of transaminases - 4 , arterial hypertension - 2), MTX-P-MP 8 pts (hair loss-1, dermatitis-1, elevation of transaminases-5, Cushing's syndrome - 1) and LEF - 5 pts (elevation of transaminases-5). At baseline all groups were comparable in their demographic, clinical and radiographic characteristics.

Conclusions: In most cases side effects were moderate or minimal. The most serious side effects, leading to the discontinuation of the therapy, were registered in LEF group. There was no withdrawal of treatment in MTX-P group. Safety profile was the same in all groups.

Disclosure of Interest: None declared

DOI: 10.1136/annrheumdis-2017-eular.6717

\section{THU0190 DERMATOLOGICAL GUIDELINES FOR MONITORING METHOTREXATE TREATMENT REDUCE DRUG-SURVIVAL COMPARED TO RHEUMATOLOGICAL GUIDELINES}

H. Bernelot Moens ${ }^{1}$, F. Busger op Vollenbroek ${ }^{1}$, R. Janssens ${ }^{2}$, C. Doggen ${ }^{3}$. ${ }^{1}$ Rheumatology \& Clinical Immunology; ${ }^{2}$ Dermatology, Ziekenhuisgroep Twente, Almelo; ${ }^{3}$ Health Technology and Services Research, University of Twente, Enschede, Netherlands

Background: Methotrexate (MTX) is widely used in the treatment of psoriasis and psoriatic arthritis (PsA). To prevent MTX-induced adverse events dermatological MTX guidelines advise a higher number and frequency of blood tests than rheumatological guidelines $(1,2)$. These differences are not based on evidence indicating a higher risk for patients with psoriasis compared to PsA.

Objectives: Compare the effects of MTX monitoring strategies by rheumatologists and dermatologists.

Methods: Patients with psoriasis or PsA in a Dutch teaching hospital. Inclusion criteria: start methotrexate (MTX) between 2006 and 2012 and scheduled follow-up by dermatologist or rheumatologist. Exclusions: incomplete availability of lab data. Start and stop dates and dosing of MTX and folic acid, reasons for withdrawal of MTX, numbers and results of laboratory tests performed for MTX safety, occurrence of any serious adverse event (SAE) were retrieved from electronic records.

Results: PsA patients used higher initial and maximum doses of MTX and folic acid, but psoriasis patients had a higher frequency of abnormal liver function tests, resulting in a striking difference in withdrawal of MTX (Table). In PsA MTX was more often withdrawn for remission, and less frequently for ineffectiveness leading to longer drug survival in the first course of treatment. There were no differences in the occurrence of SAE or death between these groups. Hospital admissions related to infection were recorded in $6(3.1 \%)$ PsA vs $4(2.1 \%)$ psoriasis patients.

Table 1. MTX dose, lab results, and reasons for withdrawal

\begin{tabular}{lcc}
\hline & Psoriasis (N=190) & Psoriatic arthritis (N=196) \\
\hline Men, N (\%) & $86(45.3)$ & $95(48.5)$ \\
Age (y), mean (SD) & $52.3(16.2)$ & $51.8(13.8)$ \\
Prior treatment with MTX & $8(4.2)$ & $39(19.9)^{\star \star}$ \\
MTX starting dose (mg/week) & $12.2(3.7)$ & $15.2(3.0)^{\star \star}$ \\
Folic acid starting dose (mg/week) & $4.9(0.7)$ & $8.4(5.6)^{\star \star}$ \\
Duration of first treatment course (months) & $19.0(19.5)$ & $34.3(30.5)^{\star \star}$ \\
Laboratory visits per treatment month & $0.62(0.81)$ & $0.50(0.34)^{\star}$ \\
Abnormal lab results per treatment month & $0.14(0.26)$ & $0.03(0.07)^{\star \star}$ \\
Abnormal lab results per laboratory visit & $0.26(0.39)$ & $0.06(0.11)^{\star \star}$ \\
Reasons for withdrawal of MTX & & \\
$\quad$ Ineffectiveness & $46(24.1 \%)$ & $31(15.8 \%)^{\star}$ \\
$\quad$ Remission & $14(7.3 \%)$ & $21(10.7 \%)$ \\
$\quad$ Abnormal laboratory result & $29(15.2 \%)$ & $8(4.1 \%)^{\star \star}$ \\
$\quad$ Drug toxicity (mild, including infection) & $47(24.6 \%)$ & $52(26.5 \%)$ \\
Serious Adverse Event & $2(1.0 \%)$ & $2(1.0 \%)$ \\
Death (not related to MTX) & $2(1.0 \%)$ & $4(2.0 \%)$ \\
Other & $22(11.6 \%)$ & $15(7.6 \%)$ \\
\hline Numbers: mean (SD), ${ }^{*} P$ value $\leq 0.05 ;{ }^{\star *} P$-value $<0.001($ Fisher's exact test),
\end{tabular}

Conclusions: Monitoring by dermatologists resulted in more abnormal liver function tests and shorter drug survival of MTX. The monitoring strategy by rheumatologists was not associated with increased SAEs. This supports the safety of current rheumatological guidelines and suggests reconsideration of a higher number of liver function tests in dermatological guidelines.

\section{References:}

[1] Menter A, Korman NJ, Elmets $C$ et al. Guidelines of care for the management of psoriasis and psoriatic arthritis. J Am Acad Dermatol. http://dx.doi.org/ 10.1016/j.jaad.2009.03.027.

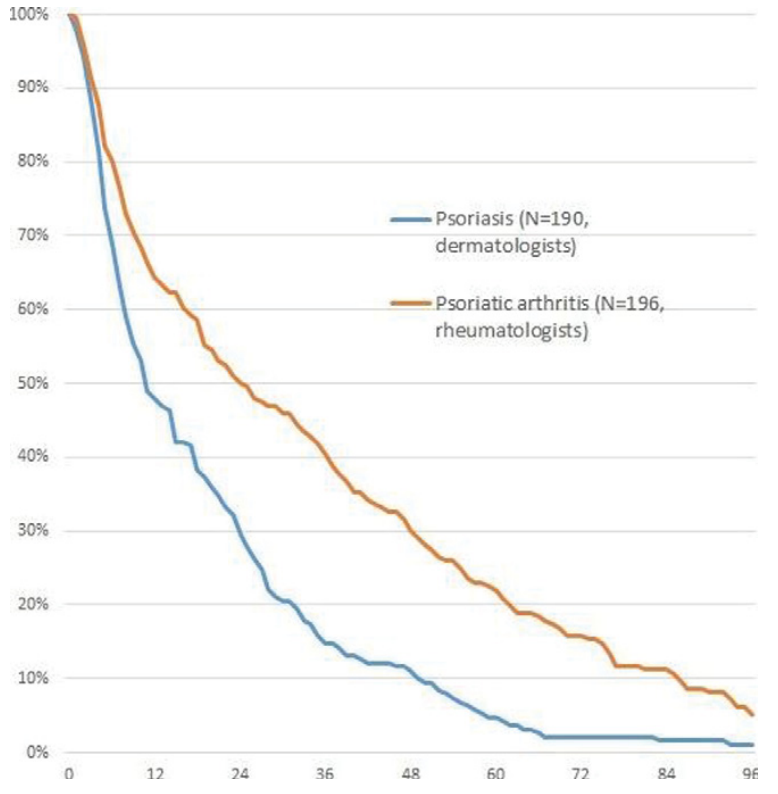

[2] Barker J, Horn EJ, Lebwohl $M$ et al. Assessment and management of methotrexate hepatotoxicity in psoriasis patients: Report from a consensus conference to evaluate current practice and identify key questions toward optimizing methotrexate use in the clinic. J Eur Acad Dermatol Venereol 2011:25:758-64.

Disclosure of Interest: None declared

DOI: 10.1136/annrheumdis-2017-eular.2524

\section{THU0191 EFFECTS OF TOFACITINIB, AN ORAL JANUS KINASE INHIBITOR, ON PATIENT-REPORTED OUTCOMES IN JAPANESE PATIENTS WITH RHEUMATOID ARTHRITIS}

H. Yamanaka ${ }^{1}$, Y. Tanaka ${ }^{2}$, T. Takeuchi ${ }^{3}$, N. Sugiyama ${ }^{4}$, T. Hirose ${ }^{4}$, N. Yoshii ${ }^{4}$, Y. Morishima ${ }^{4}$, S. Toyoizumi ${ }^{4} .{ }^{1}$ Tokyo Women's Medical University, Tokyo; ${ }^{2}$ University of Occupational and Environmental Health, Kitakyushu; ${ }^{3}$ Keio University; ${ }^{4}$ Pfizer Japan Inc, Tokyo, Japan

Background: Tofacitinib is an oral JAK inhibitor for the treatment of rheumatoid arthritis (RA). Improvements in patient-reported outcomes (PROs) have been reported in the global population of the Phase (P)2, P3 and long-term extension (LTE) tofacitinib studies.

Objectives: To explore the effect of tofacitinib on PROs in Japanese patients (pts) with RA.

Methods: In this post hoc analysis, data from Japanese pts with RA were obtained from two 12-week randomised dose-finding $\mathrm{P} 2$ studies in methotrexate (MTX) inadequate responder (IR) and DMARD-IR pts (NCT00603512/A3921039 and NCT00687193/A3921040), one 24-month P3 study in MTX-IR pts (ORAL Scan; NCT00847613/A3921044) and an open-label, LTE study in pts who completed a qualifying P2 or P3 study (NCT00661661/A3921041; completed April 2014). Pts received tofacitinib 5 or $10 \mathrm{mg}$ twice daily (BID) or placebo (PBO) (P2 and ORAL Scan; no PBO in LTE). In ORAL Scan, non-responder PBO pts advanced to tofacitinib at Month 3; all remaining pts were advanced at Month 6 . PROs included: mean change from baseline in Pt's Global Assessment of Arthritis (PtGA; visual analogue scale [VAS]), Physician's Global Assessment of Arthritis (PGA; VAS), Health Assessment Questionnaire-Disability Index (HAQ-DI), Pain (VAS), Functional Assessment of Chronic Illness Therapy - Fatigue (FACIT-F), Medical Outcomes Study (MOS) Sleep Scale and Short-Form Health Survey (SF-36) domain scores. Significance was declared for $p \leq 0.05$ for the P2 and P3 studies reported here.

Results: The analysis included 238 pts from P2 studies, 118 pts from ORAL Scan and 486 pts from the LTE study. Demographics and baseline characteristics were similar between treatment groups for all studies. In P2 studies at Week 12 , tofacitinib 5 and $10 \mathrm{mg}$ BID demonstrated significantly greater improvements from baseline vs PBO in PtGA, PGA, HAQ-DI, Pain, FACIT-F, MOS Sleep Scale and in 4 (Physical Function [PF], Role-Physical [RP], Bodily Pain [BP] and General Health [GH]) of the 8 SF-36 domain scores (Table). Significant improvements in PtGA, PGA, HAQ-DI, Pain and FACIT-F vs PBO were seen as early as Week 2. In ORAL Scan at Month 3, statistically significant improvements from baseline in PtGA, PGA, HAQ-DI and Pain were seen for both tofacitinib 5 and $10 \mathrm{mg}$ BID vs PBO (Table) and these were maintained to Month 24. Significant improvements vs $\mathrm{PBO}$ as early as Month 1 were seen for PGA (tofacitinib $10 \mathrm{mg}$ BID) and Pain (both doses). In the LTE study, mean changes from LTE study baseline in PtGA, PGA, HAQ-DI and Pain were $-32.5,-40.8,-0.5$ and -32.9 , respectively, for all tofacitinib doses at Week 2 , and $-40.7,-50.2,-0.7$ and -42.4 , respectively, at Week 168. Mean changes from baseline in SF-36 domain scores at Week 12 and 